\title{
Social Influence Aware Personalized Recommendation
}

\author{
Jinpeng Chen ${ }^{1+}$, Yihan Chen ${ }^{1}$, Wenchao Shi ${ }^{1}$, Enbo Du ${ }^{1}$, Zhipeng Huang ${ }^{1}$, Yiguang Lu ${ }^{1}$, \\ Pinguang Ying ${ }^{2}$ and Ming Zou ${ }^{3}$ \\ ${ }^{1}$ School of Software Engineering, Beijing University of Posts and Telecommunications, China \\ ${ }^{2}$ Shanghai University of International Business and Economics, China \\ ${ }^{3}$ Beihang University, China
}

\begin{abstract}
In this work, to exploit social influence in social influence aware music recommendation, we first quantify social influence. As a step further, in order to improve music recommendation by incorporating social influence, we present a factor graphic model based on social influence. Our experimental results on one real world dataset verify that our proposed approach outperforms current state-of-the-art music recommendation methods substantially.
\end{abstract}

Keywords: music recommendation, social influence, factor graphic model

\section{Introduction}

Music plays an important role in the daily lives of human beings. With the development of mobile devices and Internet technology, digital music market has been growing rapidly. Nowadays, online music services show exponential growth. At the same time, people can easily obtain hundreds of thousands of songs. However, with the increasing of the huge amount of online media content, people are facing a problem, that is how to search their desired songs from a large selection in a reasonable time. To solve this problem, music recommender systems (MRSs) have been emerging.

Social influence catches the methods in which people influence each others' thoughts, interactions, and behaviours in a social network [1]. Social influence is usually mapped in changes in social behaviour patterns in the social media. In this paper, we focus on the problem of social influence aware music recommendation, namely, exploiting social influence for music recommendation, which aims to return a list of songs for a target user. We believe that this is a natural and useful extension to the conventional music recommendation problem. However, it is challenging and crucial to predict which song a man will listen to from the sheer volume of music collection. To exploit social influence for music recommendation, we propose an approach incorporating social influence. As [2] said, users with similar interests and with frequent correlate actions have a stronger influence on each other.

The major contributions of this paper are summarized in the following.

- We focus on a new social influence aware music recommendation problem, which aims to recommend a specific list of songs for a user.

- We develop the music recommendation method that exploits social influence. Moreover, we fuse three vital features with a framework to make social influence aware music recommendation.

- We evaluate the proposed music recommendation method by comprehensive experiments on one real world dataset collected from Last.fm.

\footnotetext{
+ Corresponding author. Tel.: + 86 13811415176; fax: + 8658828012.
}

E-mail address: chenjinpeng@ @lsde.buaa.edu.cn. 


\section{Problem Definition}

DEFINITION 1. (Listening Action) We use a triple ( $u i, t \mathrm{~m}, m)$ to express that user $u i$ listens to a song $m$ at time $t m$. For the song $m$, we define all users' listening actions as the listening history $Y=\{u i, t \mathrm{~m}, m\}$ $i, t m$. Further we denote $y_{i, t m}$ as the action status of user $u i$ at time $t m$ for the given song $m$.

DEFINITION 2. (Social Influence) Social influence is usually regarded as the effect of latent predictions acquired on social network, which means that users are apt to follow their friends' behaviours [3]. The social influence propels users to take in behaviours shown by their neighbours. In other words, the stronger the relationship between two users is, the more effective the prediction is in affecting the user [6].

Problem definition. Given a music set $M$, and a user $u$, our aim is to detect the probability of user $u$ listening to music $m \in M$, defined as $P(m \mid u, M)$, then return a top-k list of music pieces with the maximum probability for $u$.

\section{Listening Action Prediction}

\subsection{Feature Definition}

In order to predict listening actions, we denote three kinds of basic features, including personal attributes, personal preferences, and social influence.

Personal Attributes (PA). We adopt five personal attributes, including age, gender, occupation, education, and the age of the account in order to model the influence of such specific factors for music recommendation.

Personal Preferences (PP). We use the method in [4] to model a use's preferences. Two features can be denoted as follows: artists and genres.

Social Influence (SI). Here, we adopted the method in [3] to capture the social influence.

\subsection{Factor Graphic Model based on Social Influence}

In this work, we present a factor graphic model based on social influence (FGMSI) to predict the listening action. A factor graph for each song $m$ is constructed. Each instance $u i \stackrel{\text { play }}{\longrightarrow} m$ can be seen as a node in the factor graph and a label $y i$ for each node is assigned, where $y i=1$ implies that user $u i$ listened to a song $m$ and $y i=0$ implies that $u i$ did not listen to this song. Each node is related to an attribute vector ${ }^{\rightarrow} x i$ and each dimension of $\vec{x} x i$ is from social influence feature and basic features denoted in the last section. Two kinds of factors are defined: the first one is the attribute factor, which refers to the posterior probability of the label $y i$ given the attribute vector $\vec{A} x i$; the second one is the correlation factor, which defines the correlation between the relationships.

Given a network $G$. a set of historical listening records $R^{u}=\left\{r_{1}^{u}, r_{2}^{u}, \ldots, r_{\left|R^{u}\right|}^{u}\right\}$, and the corresponding feature vector $X^{u}=\left\{x_{1}^{u}, x_{2}^{u}, \ldots, x_{\left|R^{u}\right|}^{u}\right\}$ with some known labelled relationships $y i=1$ (or $y i=0$ ) and some unknown labelled relationships, our aim is to predict these unknown labelled relationships.

We first define the joint distribution over $Y$ as:

$$
P(Y \mid X, G)=\Pi f\left(y_{i}, x_{i}, x_{j}\right) g\left(y_{i}, G\left(y_{i}\right)\right)
$$

where $f(y i, x i, x j)$ refers to the attribute factor and $g(y i, G(y i))$ refers to the correlation factor.

In this work, we adopt exponential-linear functions to denote these two kinds of factors. $f(y i, x i, x j)$ can be defined as:

$$
f\left(y_{i}, x_{i}, x_{j}\right)=\frac{1}{Z_{\alpha}} \exp \left\{\alpha^{\top} \phi\left(y_{i}, x_{i}, x_{j}\right)\right\}
$$

where $Z \alpha$ is a normalization factor, $\alpha$ is a weighting vector; $\varphi$ is a vector of feature functions denoted between $u i$ and $u j$ with respect to the value of $y i$; $x i$ and $x j$ are attributes associated with $u i$ and $u j$. Similarly, $g(y i, G(y i))$ can be denoted as: 


$$
g\left(y_{i}, G\left(y_{i}\right)\right)=\frac{1}{Z_{\beta}} \exp \left\{\sum_{y_{j} \in G\left(y_{i}\right)} \beta^{\top} \psi\left(y_{i}, y_{j}\right)\right\}
$$

where $\psi$ is a vector of indicator functions.

\section{Experiments}

In this section, we show various experiments to evaluate the efficiency and effectiveness of the proposed approach.

\subsection{Data set and Experimental Setup}

We collected metadata and user generated data from Last.fm via their public API. The collected dataset covers 102,112 playlists and 772,601 tags created by 216,420 active Last.fm users, who listened to $1,163,123$ unique songs from 72,601 unique albums by 80,014 unique artists categorized into 322 sub-genres from 23 genres.

In this work, we mainly concentrate on the evaluation of top results according to accuracy. We adopt precision at $\mathrm{k}(\mathrm{P} @ \mathrm{k})$, recall at $\mathrm{k}(\mathrm{R} @ \mathrm{k})$ and F-Score $(\mathrm{F} 1)$ to evaluate the recommendation performance.

The following benchmark methods are implemented for comparisons.

User-based Collaborative Filtering $(U C F)$. It assumes that similar preferences are shared by similar users.

Personality-Based Music Recommender Systems (PBMRS). It classifies the influence of integrating the target user's personality in music recommender systems [5].

\subsection{Experimental Results}

From Figure 1, we can obtain that, our proposed method is the best in terms of precision. The possible reason for these is that our proposed FGMSI I not only captures users' basic information like social relationship, personal preference, but also considers social influence, in the prediction processing. Because of the space limit, the similar analysis is shown in Figure 2 and Figure 3.

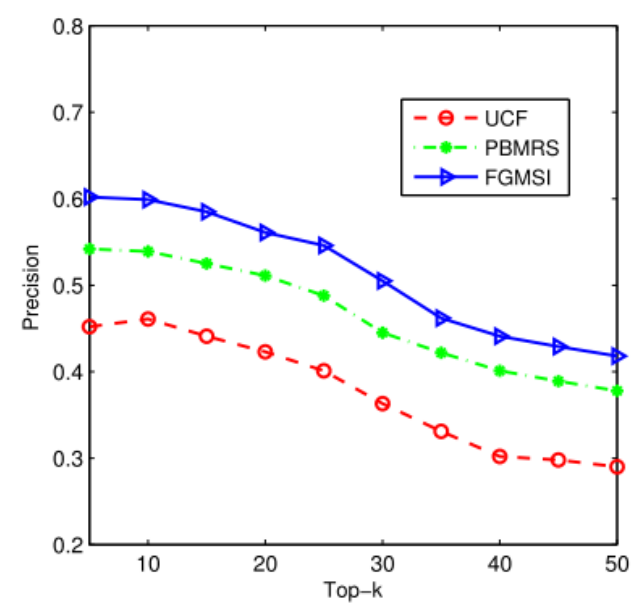

Fig. 1: Precision.

\section{Related Work}

The aim of music recommendation is to help the user easily choose the favorite music pieces from a large music archive by associating the users' social relationship, preferences with music and so on. To this end, there have already been a reasonable amount of researches on music recommendation.

In [7], authors predisposed the crawled data and observed the artists' preference and proposed a ranking based algorithm combined with these preferences. Schedl et al. detected the usage of users' demographic information in collaborative filtering [8]. In [9], Su et al. proposed a new method based on social and collaborative information that can bridge users' preferences to music effectively. In [10], Cheng et al. explored the influence of music play sequence on developing effective personalized music recommender 
systems. Cheng et al. took into account the effect of venue types on users' music preference and designed a venue-aware music recommender system [11]. In [12], a novel User-Information-Aware Music Interest Topic (UIA-MIT) model was proposed to find the potential music interest space of general users and capture the music preferences of users in different ages and genders. In [13], Schedl et al. proposed a geospatial model that leverages GPS coordinates and a cultural model that uses semantic locations for the task of music recommendation.

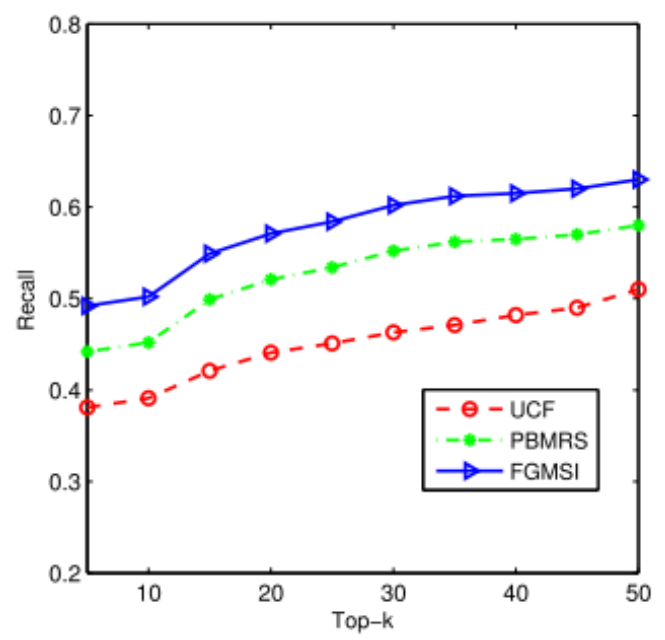

Fig. 2: Recall.

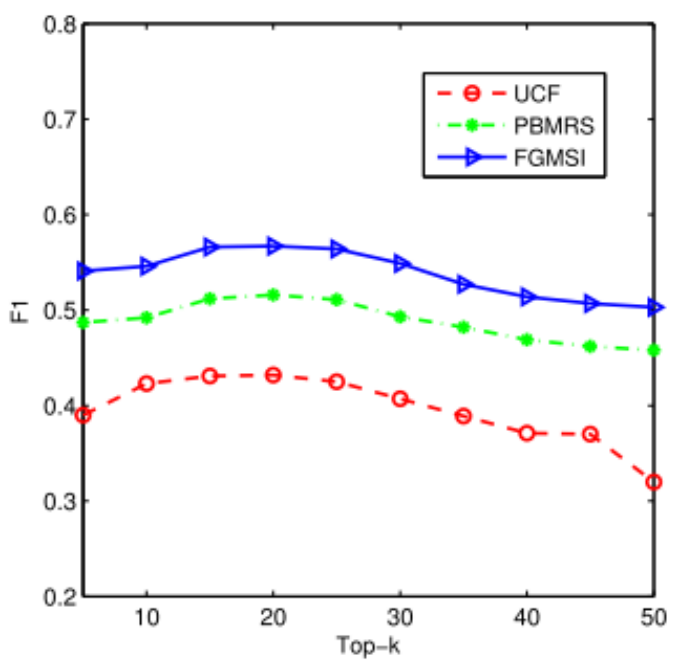

Fig. 3: F1.

\section{Conclusion}

In this work, we leverage the factor graphic model based on social influence to produce music recommendations. We conduct extensive experiments over one real-world music datasets. The experimental results show that the proposed method outperforms all the baseline methods. In the future, we will investigate more features to construct better and meaningful representations for enhancing music recommendations.

\section{Acknowledgment}

This work is supported by the National Natural Science Foundation of China under Grant No.61702043, the Fundamental Research Funds for the Central Universities under Grant No.2017RC55 and Research Innovation Fund for College Students of Beijing University of Posts and Telecommunications. 


\section{References}

[1] Zhang J, Liu B, Tang J, et al. Social influence locality for modeling retweeting behaviors[C]// International Joint Conference on Artificial Intelligence. AAAI Press, 2013:2761-2767.

[2] Schedl M, Hauger D. Tailoring Music Recommendations to Users by Considering Diversity, Mainstreaminess, and Novelty[C]// International ACM SIGIR Conference on Research and Development in Information Retrieval. ACM, 2015:947-950.

[3] Sun J, Tang J. A Survey of Models and Algorithms for Social Influence Analysis[M]//Social Network Data Analytics. Springer US, 2011:177-214.

[4] Seo Y D, Kim Y G, Lee E, et al. Personalized recommender system based on friendship strength in social network services [J]. Expert Systems with Applications, 2017, 69:135-148.

[5] Onori, Melissa, Alessandro Micarelli, and Giuseppe Sansonetti. A Comparative Analysis of Personality-Based Music Recommender Systems. In EMPIRE@ RecSys, pp.55-59. 2016.

[6] Wen Y T, Fan Y Y, Peng W C. Mining of Location-Based Social Networks for Spatio-Temporal Social Influence[M]//Advances in Knowledge Discovery and Data Mining. 2017:799-810.

[7] Lu Y, Qiao Z, Zhang P, et al. Ranking-Based Music Recommendation in Online Music Radios[C]// IEEE International Conference on Data Science in Cyberspace. IEEE, 2017:614-619.

[8] Schedl M, Hauger D, Farrahi K, et al. On the Influence of User Characteristics on Music Recommendation Algorithms[C]// European Conference on Ir Research, ECIR 2015, Proceedings. 2015:339-345.

[9] Su J H, Chang W Y, Tseng V S. Effective social content-based collaborative filtering for music recommendation [J]. Intelligent Data Analysis, 2017, 21:S195-S216.

[10] Cheng Z, Shen J, Zhu L, et al. Exploiting Music Play Sequence for Music Recommendation[C]// Twenty-Sixth International Joint Conference on Artificial Intelligence. 2017:3654-3660.

[11] Cheng Z, Shen J. On Effective Location-Aware Music Recommendation[J]. ACM Transactions on Information Systems, 2016, 34(2):1-32.

[12] Cheng Z, Shen J, Nie L, et al. Exploring User-Specific Information in Music Retrieval[C]// International ACM SIGIR Conference on Research and Development in Information Retrieval. ACM, 2017:655-664.

[13] Schedl M, Vall A, Farrahi K. User geospatial context for music recommendation in microblogs [C]// International ACM SIGIR Conference on Research \& Development in Information Retrieval. ACM, 2014:987990. 